\title{
Online yield stress measurement for real-time process control
}

\author{
F Sofrà Rheological Consulting Services Pty Ltd, Australia \\ P Bhattacharjee Rheology Solutions Pty Ltd, Australia
}

\begin{abstract}
The authors report a robust estimate of the shear yield stress of a slurry from inline measurement of a flowing slurry process stream. At present, slurry density is often used as a proxy in estimating the shear yield stress based on laboratory-scale measurements performed as a function of solids concentration. However, this approach leads to inaccuracies due to poor translation of laboratory data to operational situations and fluctuations in the sensitive yield stress-concentration dependence due to material and process variations. A robust inline measurement that directly relates to the in situ yield stress is therefore both a valuable and significant advance.

In order to provide continual, inline rheology data for process control, the OnLine Rheometer Series 1000 (OLR) was piloted for a high clay, gold process thickener underflow in a pilot pipe loop facility. Benchtop measurements of the vane yield stress, and solids concentration were performed on samples drawn from the line as the circulating liquid was progressively diluted. The measured vane yield stress was compared with the Yield Stress Index (YSI), a native function available in the software of the OLR, over a concentration (c) ranging between $49.4 w t \%<c<64.5 w t \%$. Over the concentration range studied, the vane yield stress exponentially increased with concentration and ranged between 4 and 65 Pa while the YSI values ranged between 16 and 131 units showing an identical dependence on concentration. Importantly, it was found that the YSI correlated linearly with the yield stress values measured using the vane. It follows that the YSI metric can be used to estimate the yield stress within a small factor in actual operations. This finding supports the use of the YSI as an inline control variable in mineral processing and tailings management operations; in thickening and paste preparation for example.
\end{abstract}

Keywords: rheology, yield stress, online measurement, automation

\section{Introduction}

The Lihir gold mine pressure oxidation (POX) process in Papua New Guinea experiences periodic high viscosity events in the pressure oxidation (POX) circuit with little warning, leading to reduced throughput, reduced gold recovery rates and increased operating costs.

The increase in apparent viscosity of the slurry affects the process in several deleterious ways but is not considered a process fundamental issue per se as dilution is normally an acceptable remedial action, both operationally and economically. However, there is currently no suitable method for monitoring the viscosity of feed to provide enough advance warning of a high viscosity event to allow mitigation of downstream effects. As such, based on rheology testwork undertaken by Rheological Consulting Services Pty Ltd (RCS), inline rheology measurement was recommended to provide warning of and in helping the mitigation of a viscosity related process issues. For onsite measurement as a precursor to management and control of slurry rheology, RCS recommended investigation of Rheology Solutions Pty Ltd's OnLine Rheometer (OLR) for measurement of the rheology of the viscous, particulate slurry inline and in real time.

The goal of the work was to show a mechanism for slurry monitoring and measurement to provide advance warning of a high viscosity event and to allow remedial action to be implemented in a timely manner. This work describes the proof of concept investigation conducted to show that the OLR is a suitable instrument and includes OLR validation in a pipe loop, inclusive of reconciliation of OLR data output with rheological 
parameters. Though the current work describes a feed stream investigation, the results are equally applicable to process and tailings streams.

\section{OnLine Rheometer}

\subsection{Principle of operation}

Traditionally, yield stress has been measured at vanishingly small shear rates with the focus on capturing the dynamics leading to the first rapid increase in the rate of strain (Sofrà \& Boger 2002; Sofrà et al. 2015). This approach has been fruitful over the years and several alterations of the original concepts have been proposed to include linear and non-linear viscoelasticity that arise prior to yielding. However, bias toward low shear rates precludes any direct application of the resultant techniques to in situ, flowing stream measurements for industrial application. This is primarily because the microstructure in flow fields encountered in practice have a complicated deformation history, while traditional techniques for measuring yield stress favour carefully constructed, near-quiescent conditions as a starting point.

The measurement principle that underpins the operation of the OLR is oscillatory squeeze flow. It has been shown (Field et al. 1996; Bell et al. 2006; Koningsberg et al. 2013) that in controlled conditions, the technique allows for the measurement of viscoelastic properties under flow conditions and that these measurements are identical to those measured in standard (static) laboratory experiments. By controlling the flow condition through the pipe housing the rheometer, the degree of shear flow imparted can be regulated. In this limit, the measuring technique resembles techniques like superposition rheology (Vermant et al. 1998), and the results are arguably more relevant to processing operations.

Superposition techniques are well established in the rheological literature and can in fact pick up subtle changes brought about in the microstructure by flow. It has been demonstrated that the microstructure can evolve in a spatially selective way during flow (Kim et al. 2014; Varadan \& Solomon 2001; Hoekstra et al. 2005,2003 ) and impact mechanical properties (Colombo et al. 2017). It has also been shown that shear flow changes the relaxation time of aggregated slurries by progressively reducing their size without changing the internal structure (Colombo et al. 2017), implying that the information on the forces that hold the structures together are still available in a flowing system, albeit in a more distributed fashion. Also, the frequency spectrum used can be utilised to probe the hydrodynamic domains in which local forces dominate (Schroyen et al. 2017).

Since these forces provide the resistance leading to the existence of a yield stress, the techniques used in the OLR can quantify the forces (thereby the yield stress) even in a flowing stream. The accuracy of the measurements obviously depends on the conditioning of the stream that enters the measuring cavity and on the oscillation frequency spectrum chosen. Given the particle size distribution, the frequency range can be selected for a given slurry that best characterises the property of interest. For the pilot project discussed here, these aspects were tailored to focus on the yield stress of the slurry, during the configuration of the OLR.

\subsection{Rheometer description}

The OLR, shown in Figure 1, is a process rheometer that can be fitted in a pipeline to measure the flow properties of conveyed fluids without the need for manual sampling. It involves two diametrically opposed parallel plates, maintained at a predetermined gap in which the sample of interest is sandwiched during measurements. Both plates are serrated to minimise slip. At the rest (home) position, the top plate is about $5 \mathrm{~cm}$ away from the bottom plate, with the slurry flowing between the plates. When measurement commences, the top plate moves down to within a predetermined distance from the bottom plate. During the measurement cycle, the top plate moves up and down periodically at a small amplitude over a range of chosen frequencies $(\omega)$ and compresses/decompresses the sample. Typically, the frequency range is between 1 and $100 \mathrm{~Hz}$, and the amplitude is about one micron for a gap set at $0.5 \mathrm{~mm}$. The bottom plate is attached to a force transducer and records the force. Using the deformation and the force information, the OLR 
provides a data output of the viscosity as function of shear rate for the range of frequencies available. The ability to generate a full viscosity curve distinguishes the OLR from typical inline viscosity measurement instruments like the Coriolis flow meter which provide a single viscosity value at a very high shear rate. When the measurement cycle is completed, the top plate retracts back to the home position and the flow refreshes the sample between the plate.

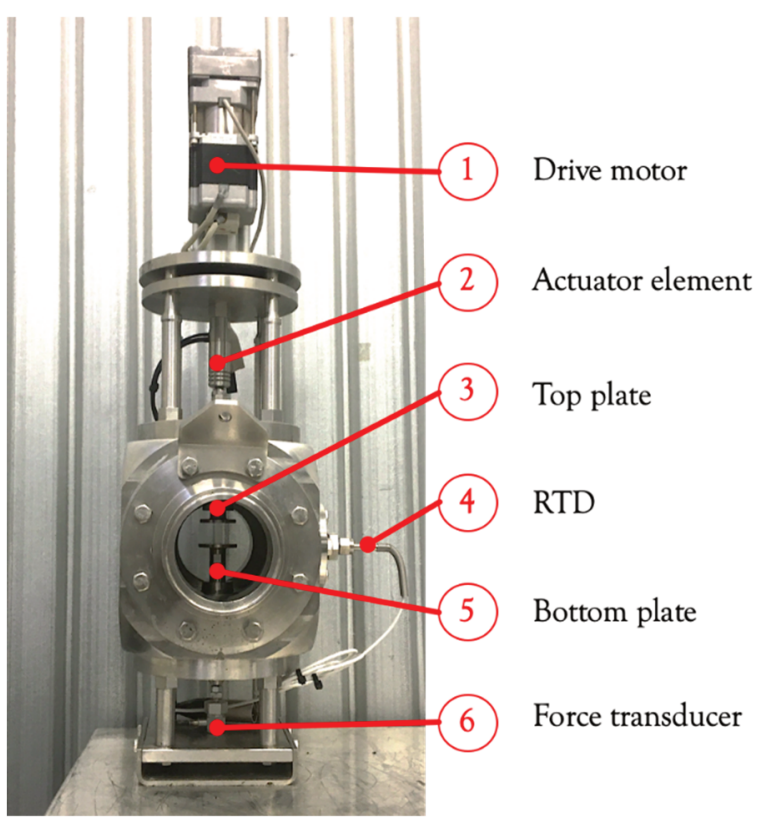

(a)

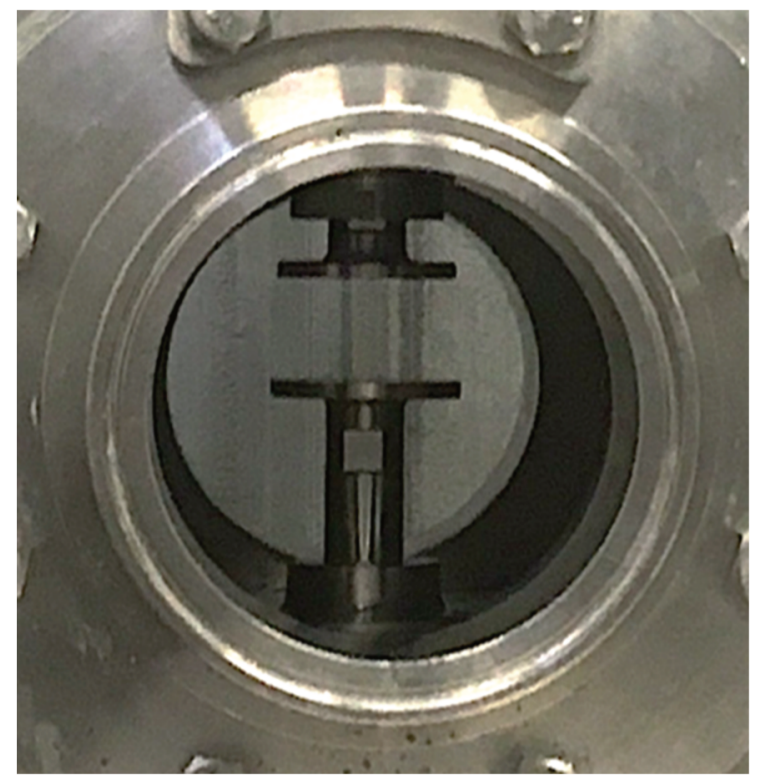

(b)

\section{Figure 1 (a) OnLine Rheometer schematic; (b) Photograph of internal plates}

The OLR can be used in an 'inline mode' where it is directly installed in the pipe conveying the slurry or in an 'online mode', where it is positioned in a side-stream separated from the main flow using an isolation valve. In this study, the OLR was trialled in the 'inline mode' in a pipe loop using Lihir thickener underflow slurry shipped to Melbourne.

The purpose of this proof of concept study was to assess the suitability of the OLR as an in situ rheology sensor, that can serve as an early warning and yield stress quantification system for detection and quantification (by proxy) of viscosity excursions.

The study involved in-tandem pipe loop testing of the thickener underflow slurry in the OLR and benchtop rheology testing.

The critical performance attributes (CPA) evaluated were:

CPA 1: Capability of the OLR for measuring the dense particulate slurry of interest, inline and in real time.

CPA 2: Possibility of using the measurements of the OLR for estimating the yield stress (or a surrogate) of the slurries.

CPA 3: Sensitivity of the OLR measurements to concentration changes.

CPA 4: Mechanical reliability over the duty cycle that would be needed for the instrument to succeed in the field. 


\section{$3 \quad$ Investigation method}

\subsection{Sample preparation}

One 1,000 L intermediate bulk container (IBC) full of thickener underflow was collected from Lihir for this testwork, along with site process water. The slurry provided was representative of material encountered during a high viscosity event. On receipt, the settled solids from the IBC were combined with some of the process water to create a high solids concentration slurry (approximately $65 \mathrm{wt} \%$, which is higher than would be anticipated during normal plant operations) for rheological and pipe loop testing. During operation, the slurry was diluted by adding process water into the mixing tank at progressive intervals to assess OLR operation throughout a range of concentrations and, therefore, rheological characteristics.

Following each dilution, the OLR outputs were monitored. Once stable flow rate and rheology data were recorded, a sample was collected for (offline) yield stress and viscosity measurement using a Haake VT550 rheometer. All samples were fully sheared (checked by monitoring yield stress) prior to OLR and benchtop testing.

Material densities were determined gravimetrically. Particle size distribution (PSD) measurements were conducted using a Malvern Mastersizer 3000 laser diffraction particle size analyser with a particle size measurement range of 0.01 to $3,500 \mu \mathrm{m}$.

\subsection{Vane yield stress measurements}

For comparison with OLR results from pipe loop testing, the yield stress of the sample was independently measured using the industry accepted vane-shear method (Nguyen \& Boger 1983, 1985, 1992) with a Haake VT550 rheometer. Results were modelled using a power law fitting function.

\subsection{Pipe loop}

The pipe loop and OLR were run for several days with very frequent data taking events to demonstrate that the instrument is reliable and robust.

A schematic diagram of the pipe loop used in this work is shown in Figure 2. The loop comprised a $350 \mathrm{~L}$ stainless steel (unbaffled) tank (4) equipped with a paddle agitator and a thermal jacket. A mono pump (5) was placed under the tank to convey the material through the $70 \mathrm{~mm}$ id stainless steel pipes that constitute the loop. The main measuring part of the loop (A-B-C-D-E-F-G) was instrumented with a Coriolis flow meter (2) (E\&H Proline Promass, Endress Hauser, Switzerland), capable of measuring the temperature, the flow rate, the density and the viscosity, and a inline viscometer (Viscoscope 4450, Marimex, Canada) (6), providing single-point viscosity, density and temperature. The main loop also had a bank of pressure transducers (8) (for pressures of one pascal or higher) and the OLR (OnLine Rheometer Series 1000, Rheology Solutions, Australia) (1) which measures the storage and loss moduli over a frequency range of 1 to $100 \mathrm{~Hz}$ and provided a flow curve for the rheology of the slurry in the loop. A photograph of the section of the loop containing the OLR is shown in Figure 3. In addition, this part of the loop is equipped with an inspection window (7) to monitor any visible settling of the slurry that is being conveyed. The loop is equipped with several control valves (9) that can isolate parts of the loop. For instance, at times the loop section A-B-G-F was only used to recirculate the slurry for mixing and homogenisation purposes.

During the project, the slurry flow rate was maintained around $4,750 \% .250 \mathrm{kgs} / \mathrm{h}\left(4,280 \mathrm{~m}^{3} / \mathrm{h}\right.$ to $6,550 \mathrm{~m}^{3} / \mathrm{h}$ at 49 and $64 \mathrm{wt} \%$ solids concentration, respectively). The sample was pumped into the bulk tank at the beginning of the testing and was periodically diluted by addition of water as the measurements progressed. The measurements were completed over a period of seven days. 


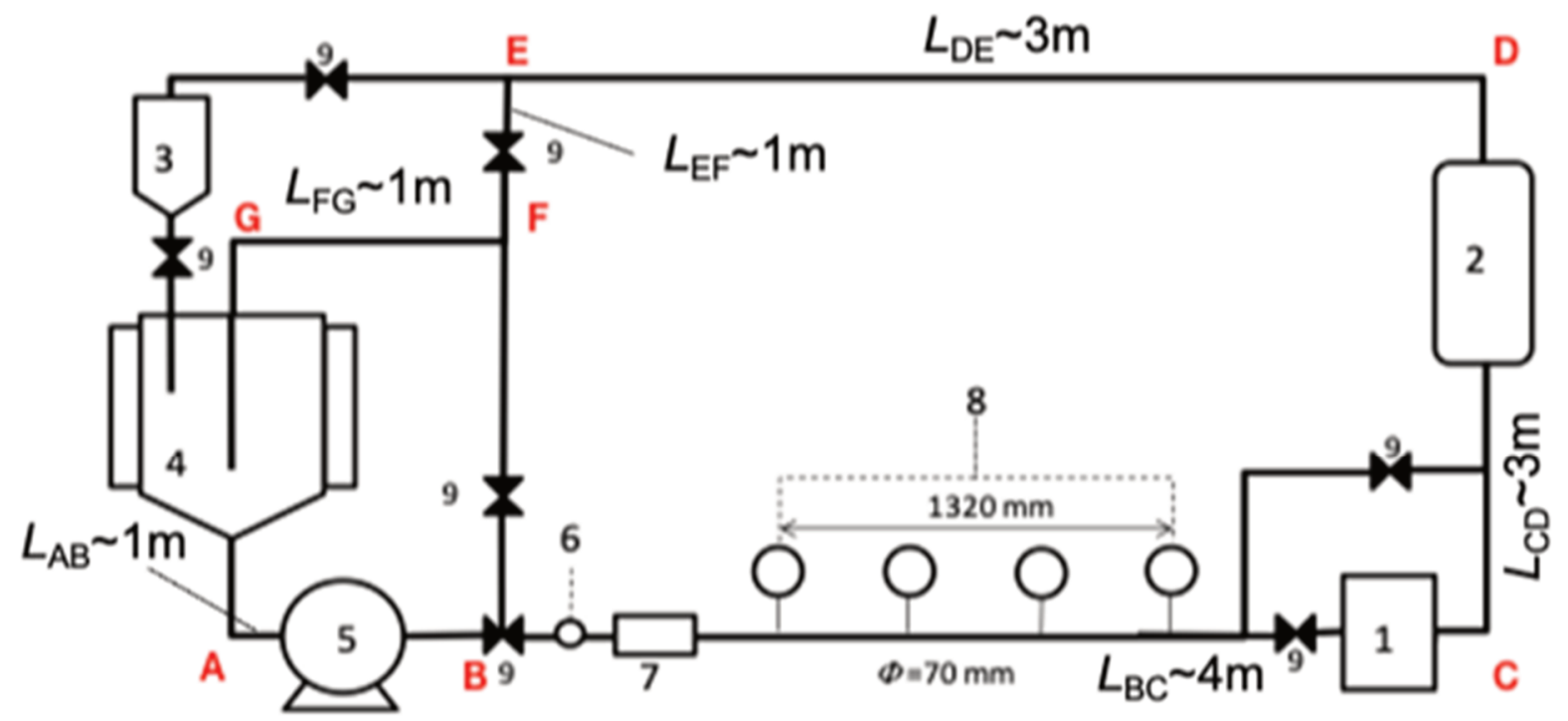
1: OLR
4: Bulk tank
7: Inspection window
2: Flowmeter
5: Mono pump
8: Pressure transducers
3: Measuring tank
6: Online Viscometer
9: Valves

Figure 2 Schematic of the pipe loop with OLR

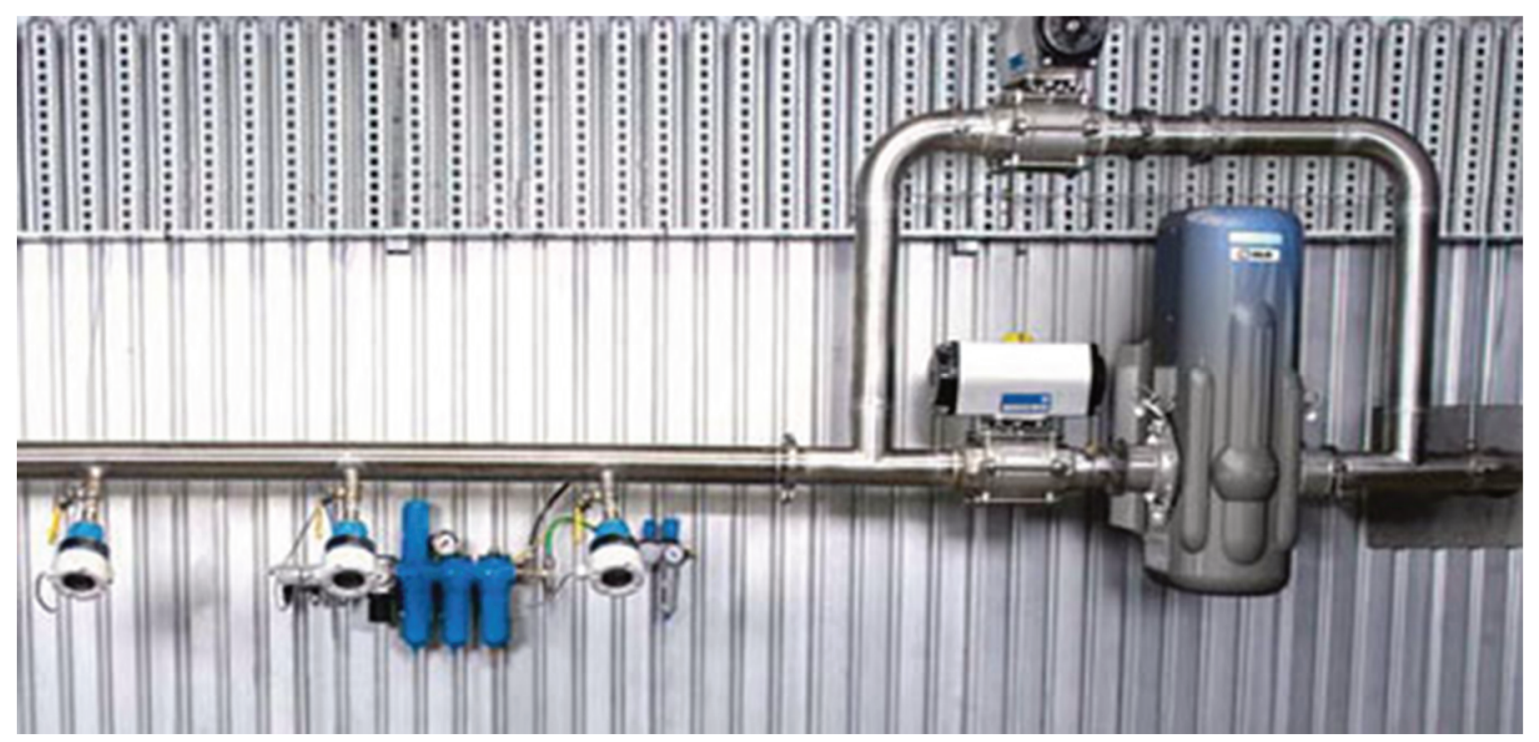

Figure 3 Photograph of the OLR section of the pipe loop

\section{Results and critical performance attribute assessment}

\subsection{Sample baseline characterisation}

The average solids specific gravity (SG) of the numerous samples of the IBC slurry tested was 2.96 , which is within the normal reported range.

The cumulative PSD of the underflow sample is shown in Figure 4. Current results, combined with other findings obtained by the author in 2018 , indicated that the sample used in this study was representative of a high viscosity (high clay) ore. 


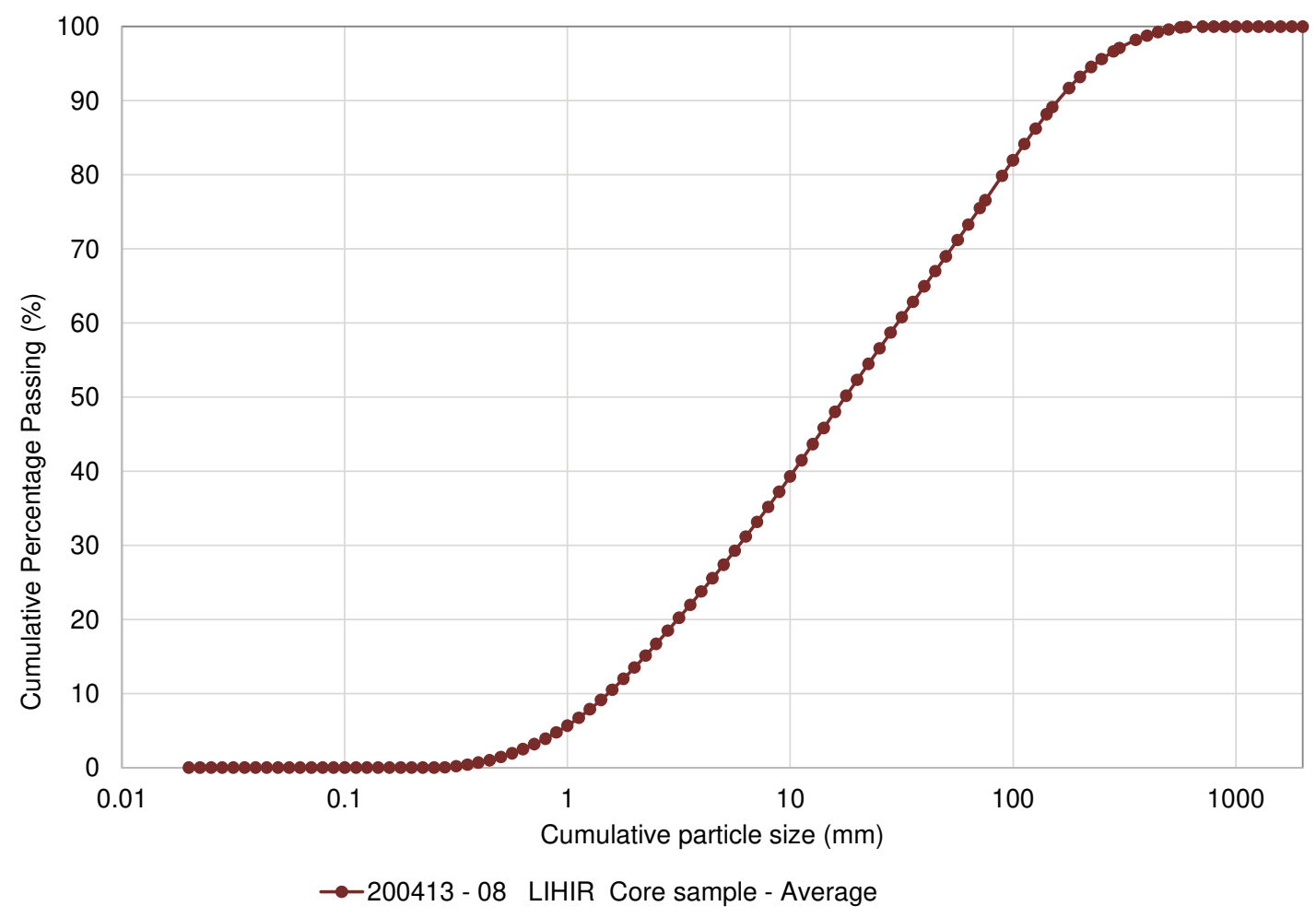

Figure 4 Cumulative particle size distribution for Lihir thickener underflow

The shear yield stress profiles of the thickener underflow samples tested are given in Figure 5 . These vane yield stress data are obtained for samples taken directly from the pipe loop as the OLR was being tested, and also taken from the stock sample that had not been subjected to sustained flow through the loop. Data from both sub-samples were identical, indicating no shear history effects during pipe loop testing.

Testing coarser slurries than that investigated in this work is possible if the force on the bottom plate can be measured reliably, and the gap between the plates is not clogged by the particle size.

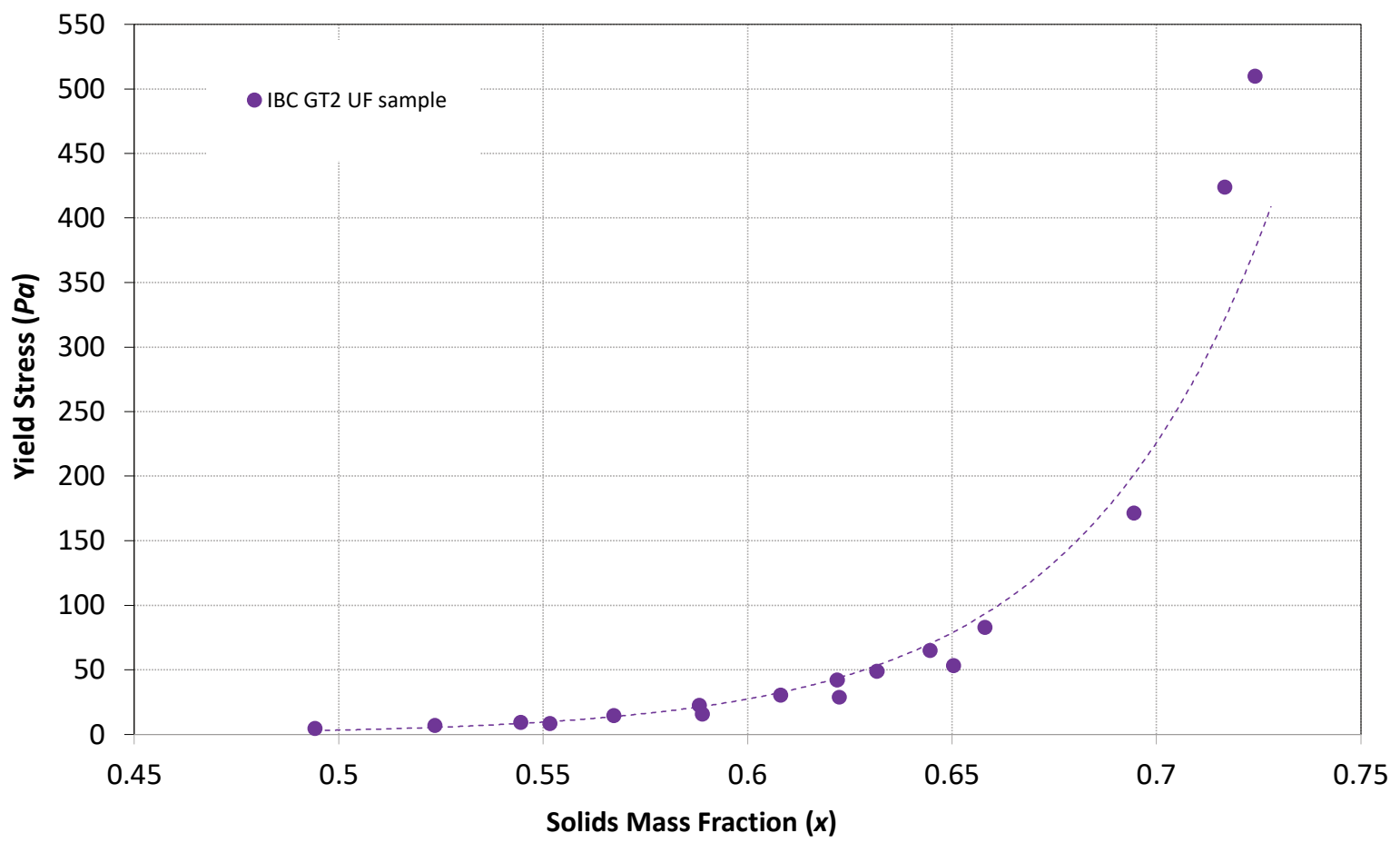

Figure 5 Lihir thickener underflow vane yield stress as a function of solids concentration 


\subsection{CPA1 - capability of the OLR for measuring the dense particulate slurry of interest, inline and in real time}

Figure 6 plots a complex viscosity parameter (Eta* or $\eta^{*}$ ) at different frequencies for the ore in the pipeline across a series of dilution events. The data indicates that the OLR was able to detect small changes in rheology as a function of a very small change in solids concentration over a range well beyond the range of solids concentrations and rheological behaviours observed in full-scale operation. The step changes in $\eta^{*}$ correlate exactly with changes in solids concentration. For each dilution level, the OLR response was rapid. Stability of readings was achieved quickly and maintained prior to the subsequent dilution.

The variation in $\eta^{*}$ (the complex viscosity) as a function of frequency $(11,46$ and $77 \mathrm{~Hz})$ shows that the slurry is shear thinning (i.e. the viscosity reduces as frequency increases) and the slurry will have a lower viscosity at higher shear (or flow) rates. This observation is expected but confirmed none the less by shear rheology results (measured but not reported here).

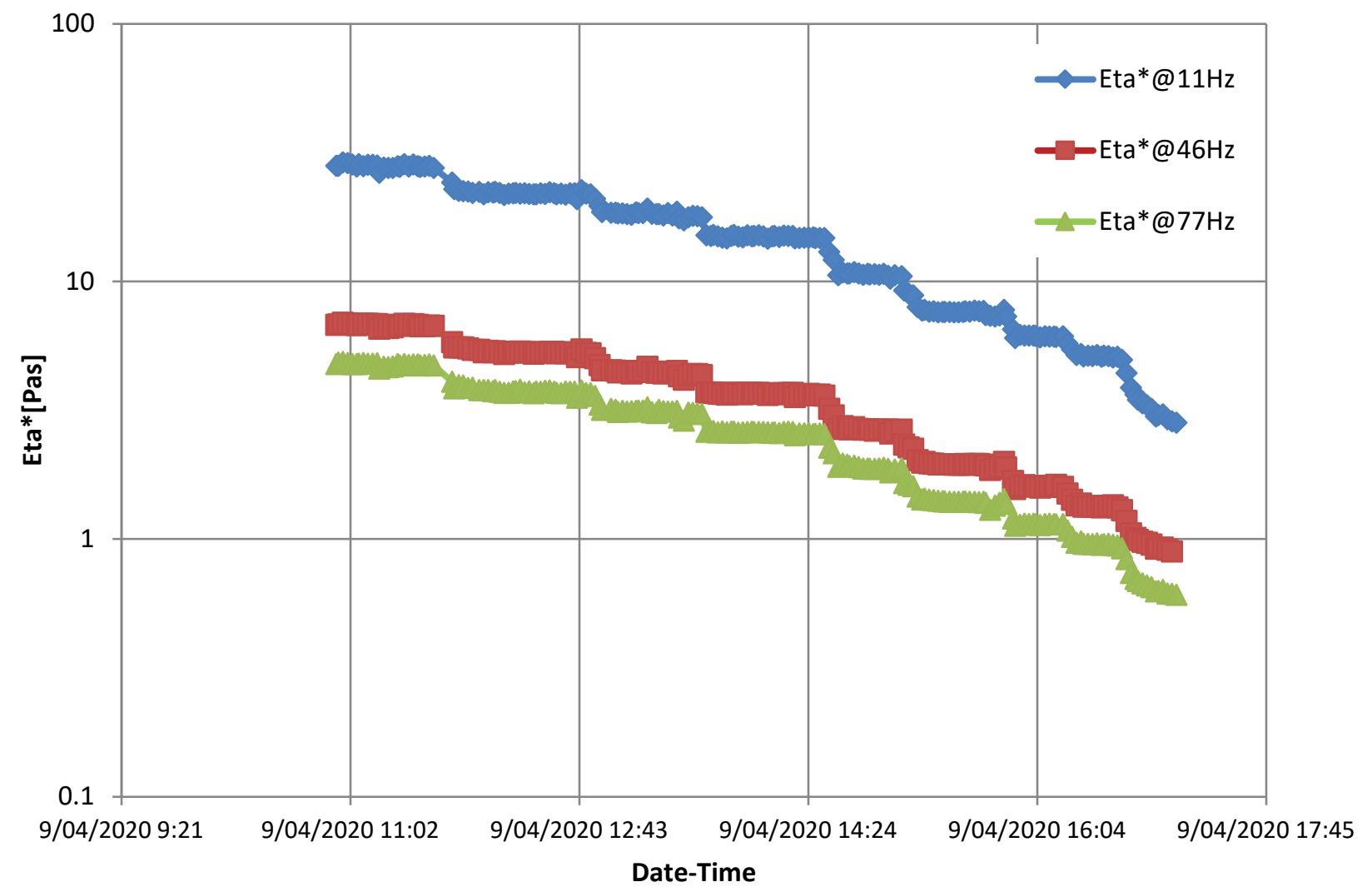

Figure 6 Pipe loop OLR data showing the complex viscosity (Eta* or $\eta^{*}$ ) as a function of time for different OLR frequencies. Step changes in data reflect dilution events

\subsection{CPA 2 - possibility of using the measurements of the OLR for estimating the yield stress of the liquids as measured by standard laboratory tests}

Figure 7 shows the relationship between the measured vane yield stress and a value (Yield Stress Index, YSI) calculated from the OLR data. The plot confirms a linear relationship between the measured vane yield stress and the calculated YSI. The relationship is consistent through the range of solids concentrations tested in the pipe loop OLR and of operational interest.

The existence of a consistent relationship between the measured vane yield stress and the calculated YSI is the most valuable result of the current study and confirms the validity of OLR measurements for determination of slurry rheological behaviour. 
Correlation with flow model outputs from viscosity measurements using rotational rheometry has been positive in terms of yield stress prediction, and flow model viscosity correlation is possible with adjustment of OLR settings. This work is outside the scope of this paper.

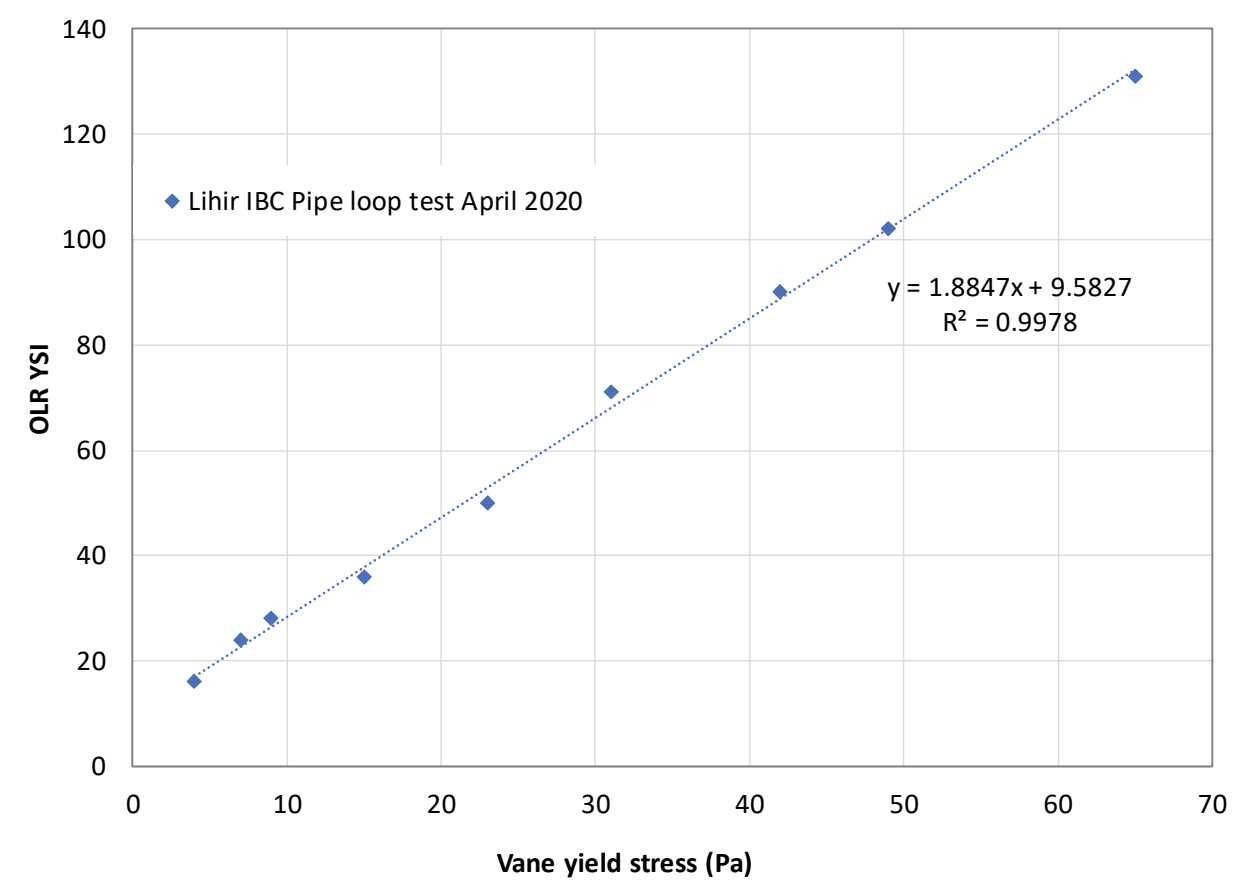

Figure 7 Direct comparison of pipe loop OLR YSI against vane yield stress, measured on the same samples

\subsection{CPA 3 - sensitivity of the OLR measurements to dilution events}

Figure 8 shows results for the $11 \mathrm{~Hz}$ dataset along with the directly measured vane yield stress results and the calculated YSI. Changes in the complex viscosity and calculated YSI for each dilution are consistently measurable by the OLR and are consistent with the changes in the directly measured vane yield stress.

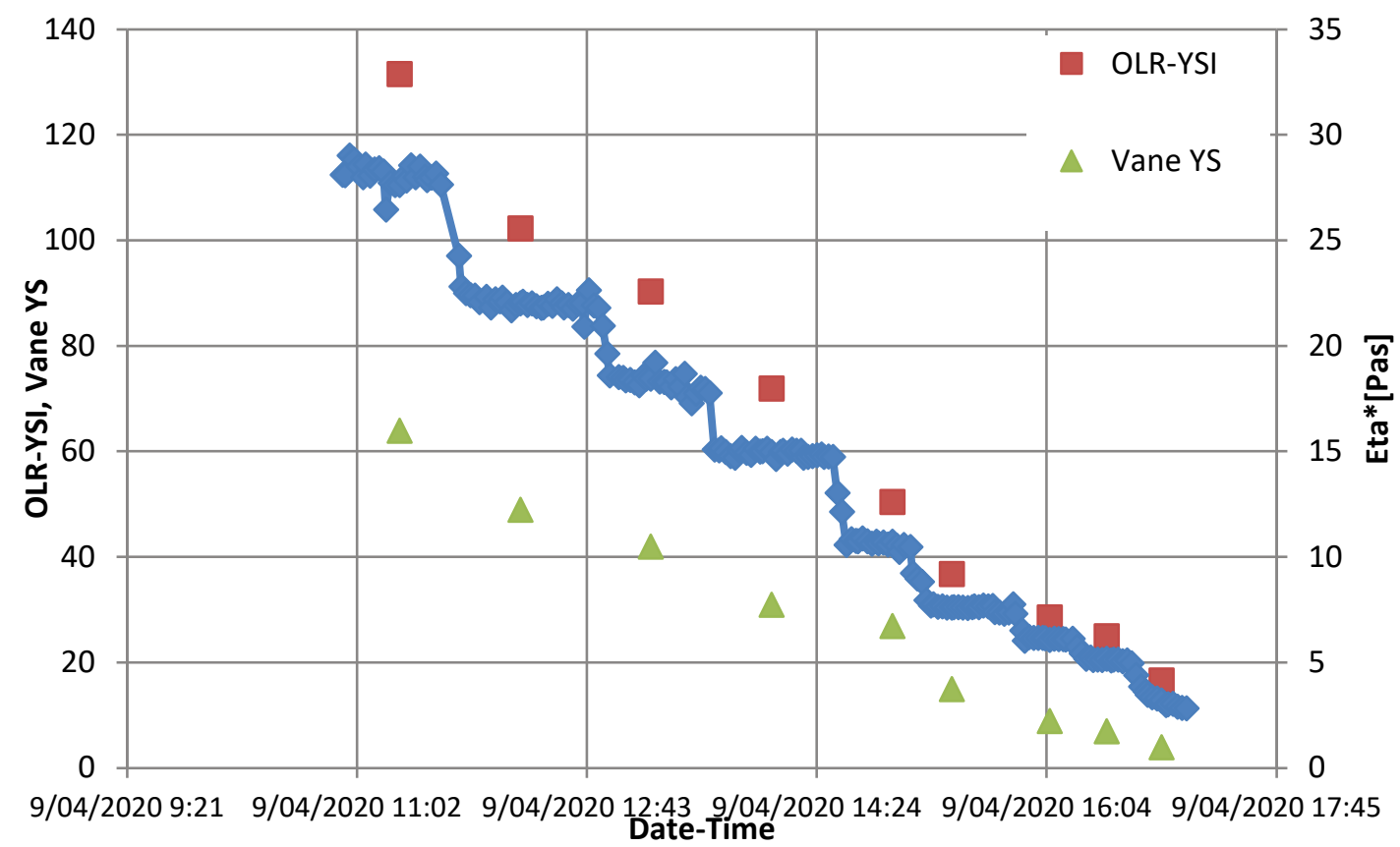

Figure 8 Pipe loop OLR data showing calculated OLR YSI and independently measured vane yield stress. Step changes reflect dilution events 


\subsection{CPA 4 - mechanical reliability over the duty cycle that would be needed for the instrument to succeed in the field}

During the work, the OLR was programmed to collect data every three to five minutes and complete around 5,000 measurement cycles overall. To keep this number in context, for a six-month service cycle, this is representative of hourly or more frequent measurement.

Following the tests, the OLR was dismantled and inspected for damage. Figures 9a and 9b show the condition of the flow cell and the bottom connection after dismantling. Some deposits were noticeable. Figures $9 c$ and $9 \mathrm{~d}$ are photographs of the same parts after flushing with water. The deposits could be completely removed, indicating that if a clean-in-place routine using water was used periodically, the progressive build-up of deposits can be avoided.
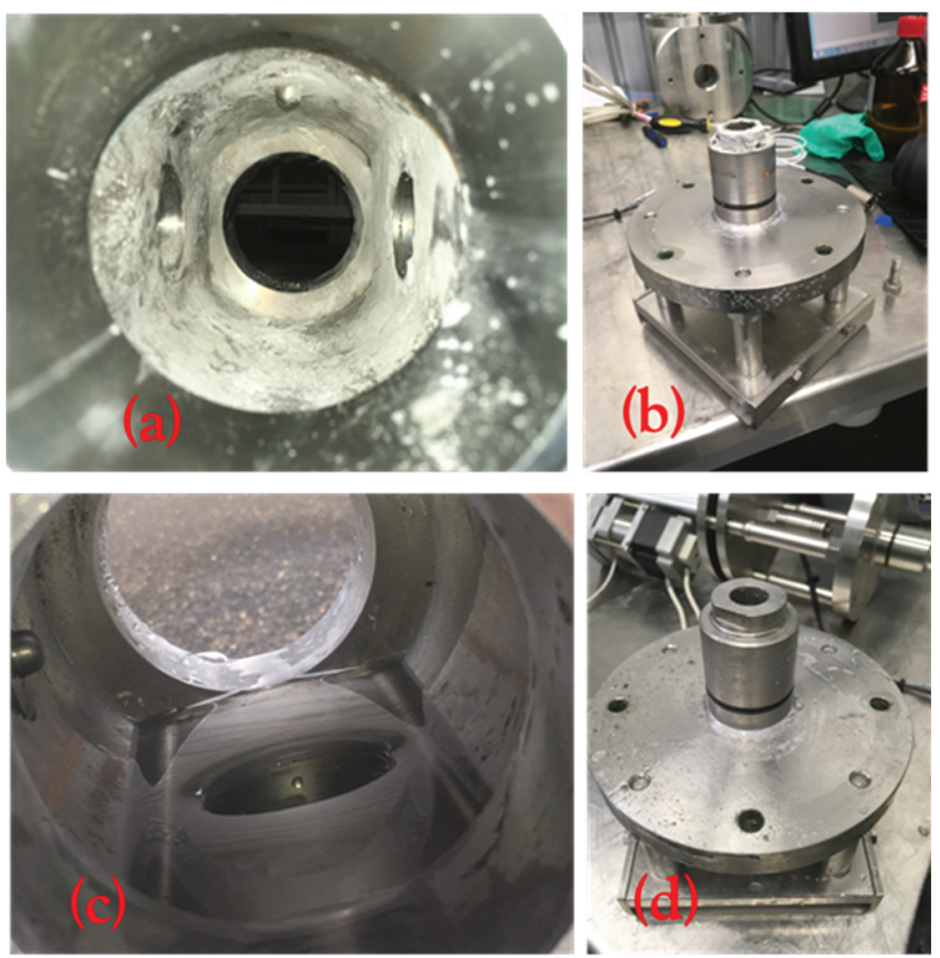

Figure 9 Condition of the flow cell and the bottom connection after dismantling

Figure 10a shows the top seal through which the actuator shaft attached to the top plate reciprocates after it has been extracted from the OLR. Some deposits are noticed in the top seal as well, as is expected. However, these deposits were easily removed by washing with water, as is shown in Figure $10 \mathrm{~b}$.
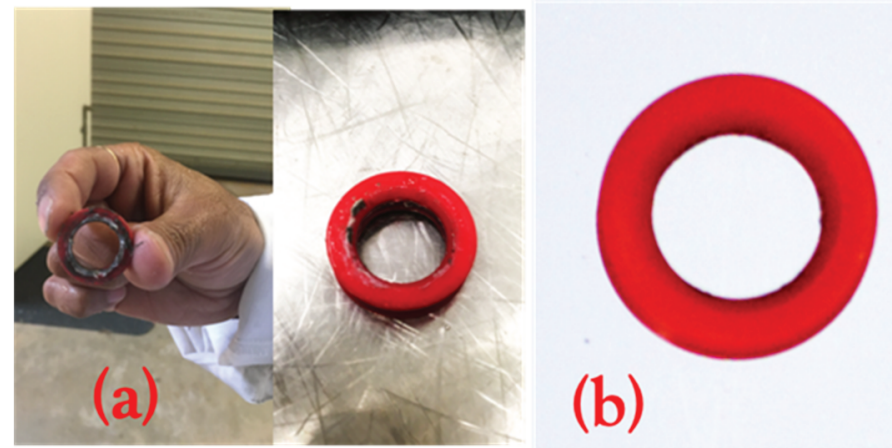

(b)

Figure 10 (a) Top seal through which the actuator shaft attached to the top plate reciprocates after it has been extracted from the OLR; (b) After washing with water 
Both the bottom seal (attached to the force sensor shaft) and the top seal, attached to the actuator shaft, were further inspected for damage using high contrast imaging, with an edge detection filter. These results are shown in Figure 11. While no aberration of the inner circumference (sealing-edge) was noticed in the bottom seal, some deviations were noticed in the top seal. This, however, should be considered in the context that the duty cycle used in the trials, and the emergence of the aberrations in the top seal is expected. Seal checking and replacement in service cycles would be able to pick up these problems before they develop sufficiently to affect operation of the OLR.
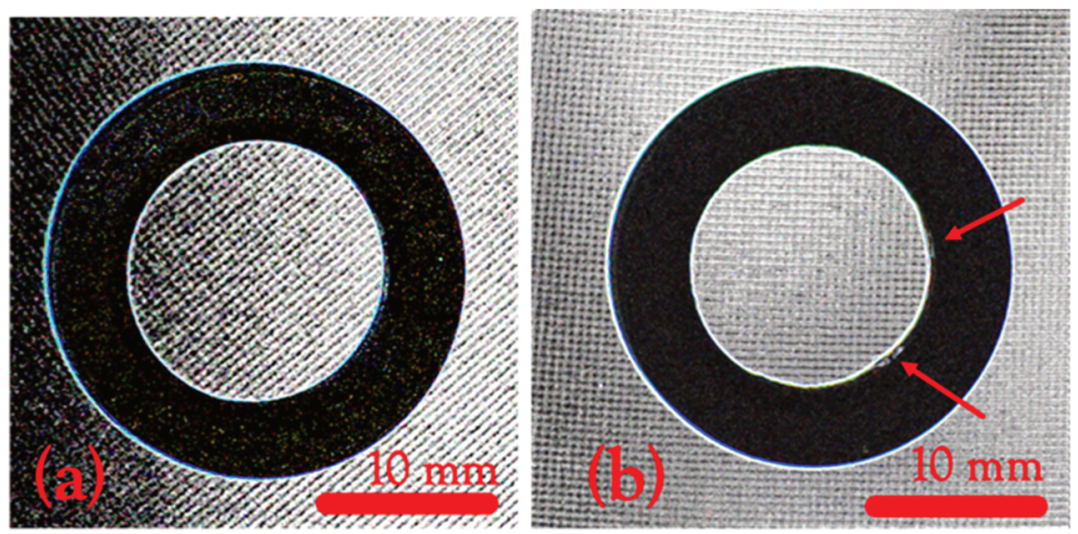

Figure 11 (a) Bottom seal (attached to the force sensor shaft); (b) The top seal attached to the actuator shaft inspected using high contrast microscopy, shown with an edge detection filter

Based on investigation of the critical mechanical components of the OLR after a series of measurements, it is expected that a half-yearly maintenance schedule would be adequate for operational purposes.

\section{Conclusion}

The OLR detected small changes in material flow behaviour in response to very small changes in solids concentration over a range of solids well in excess observed in full-scale operation. The measurements made by the OLR could be represented by a metric called the 'OnLine Rheometer Yield Stress Index'. This parameter correlated linearly with the industry standard yield stress, measured in the laboratory using a vane geometry.

The OLR measurements rapidly and accurately sensed the controlled-dilution events that were initiated at different points in time, and the yield stress of the diluted slurry could be accurately represented by the OLR YSI metric.

Investigation of the critical mechanical components of the OLR, after subjecting it to duty cycles expected in actual operations (albeit under controlled conditions), revealed no evidence of substantial wear that could lead to a catastrophic failure of the instrument in the field. It is expected that a half-yearly maintenance schedule would be adequate for operational purposes.

\section{Acknowledgement}

The authors gratefully acknowledge Raul Cavalida for his diligent and thorough work throughout the laboratory and pilot testing, and Professor Peter Scales and Pat Griffin for technical and engineering support. The authors also acknowledge Newcrest Limited for the company's support, and particular thanks John O'Callaghan for company and management support, and to Eleanor Drake for the unenviable task of sample provision.

\section{References}

Bell, D, Binding, D \& Walters, K 2006, 'The oscillatory squeeze flow rheometer: comprehensive theory and a new experimental facility', Rheologica Acta, vol. 46, no. 1, pp. 111-121. 
Colombo, G, Kim, S, Schweizer, T, Schroyen, B, Clasen, C, Mewis, J \& Vermant, J 2017, 'Superposition rheology and anisotropy in rheological properties of sheared colloidal gels', Journal of Rheology, vol. 61, pp. 5, pp. 1035-1048.

Field, JS, Swain, MV \& Phan-Thien, N 1996, 'An experimental investigation of the use of random squeezing to determine the complex modulus of viscoelastic fluids', Journal of Non-Newtonian Fluid Mechanics, vol. 65, pp. 177-194.

Hoekstra, H, Mewis, J Narayanan, T \& Vermant, J 2005, 'Multi length scale analysis of the microstructure in sticky sphere dispersions during shear flow', Langmuir, vol. 21, pp. 11017-11025.

Hoekstra, H, Vermant, J, Mewis, J \& Fuller, GG 2003,'Flow-induced anisotropy and reversible aggregation in two-dimensional suspensions', Langmuir, vol. 19, pp. 9134-9141.

Kim, M, Eberle, APR, Gurnon, AK, Porcar, L \& Wagner, NJ 2014, 'The microstructure and rheology of a model, thixotropic nanoparticle gel under steady shear and large amplitude oscillatory shear (LAOS)', Journal of Rheology, vol. 58, pp. 1301-1328.

Konigsberg, D, Nicholson, T, Halley, P, Kealy, T \& Bhattacharjee, P 2013, 'Online process rheometry using oscillatory squeeze flow', Applied Rheology, vol. 23, no. 3, https://doi.org/10.3933/applrheol-23-35688

Nguyen, QD \& Boger, DV 1983, 'Yield stress measurement for concentrated suspensions', Journal of Rheology, vol. 27, no. 4, pp. 321-349.

Nguyen, QD \& Boger, DV 1985, 'Direct yield stress measurement with the vane method'. Journal of Rheology, vol. 29, no. 3, pp. 335-347.

Nguyen, QD \& Boger, DV 1992,' Measuring the flow properties of yield stress fluids', Annual Review of Fluid Mechanics, vol. 24, no. 1, pp. 47-88.

Schroyen, B, Swan, JW, Van Puyvelde, P \& Vermant, J 2017, 'Quantifying the dispersion quality of partially aggregated colloidal dispersions by high frequency rheology', Soft Matter, vol. 13, no. 43, pp. 7897-7906.

Sofrà, F \& Boger, DV 2002, 'Environmental rheology for waste minimisation in the minerals industry', The Chemical Engineering Journal, vol. 86, pp. 319-330.

Sofrà, F, Boger, DV \& Scales, PJ 2015, 'Rheological concepts', in RJ Jewel \& AB Fourie (eds), Paste and Thickened Tailings - A Guide, 3rd edn., Australian Centre for Geomechanics, Perth.

Varadan, P \& Solomon, MJ 2001, 'Shear-induced microstructural evolution of thermoreversible colloidal gel', Langmuir, vol. 17, pp. 2918-2929.

Vermant, J, Walker, L, Moldenaers, P \& Mewis, J 1998, 'Orthogonal versus parallel superposition measurements', Journal of Non-Newtonian Fluid Mechanics, vol. 79, pp. 173-189. 
